\title{
MITTEILUNGEN DER OBVSG
}

\section{von Mathis Kronschläger}

Zusammenfassung: In den „Mitteilungen der OBVSG“ gibt die Österreichische Bibliothekenverbund und Service GmbH einen Überblick über aktuelle Ereignisse aus dem Verbund und der Verbundzentrale.

Schlagwörter: Österreichischer Bibliothekenverbund, OBV, Österreichische Bibliothekenverbund und Service GmbH, OBVSG, Verbundsuchmaschine, Primo, Alma, Digitaler Assistent, Sacherschließung, Langzeitarchivierung, Gemeinsame Normdatei, GND

\section{COMMUNICATIONS OF THE AUSTRIAN LIBRARY NETWORK AND SERVICES LTD (OBVSG)}

Abstract: The Austrian Library Network and Services Ltd (OBVSG) provides services for Austrian research and administrative libraries and is the library network's headquarters. "Communications of the OBVSG" inform about current and upcoming events concerning OBVSG and the Austrian Library Network.

Keywords: Austrian Library Network, OBV, Austrian Library Network and Services Ltd, OBVSG, Primo, Alma, subject indexing, digital preservation, Integrated Authority File, GND

\section{Inhalt}

1. Für jeden Anlass das passende Layout - Die Verbundsuchmaschine im neuen Design

2. Grüne Welle für Alma - Aktuelles zur Alma-Implementierung im OBV

3. Wissen vermehrt sich, wenn man es teilt - Der Digitale Assistent startet im Verbund

4. Save the Data - Ausschreibung eines konsortialen Systems zur digitalen Langzeitarchivierung

5. GND-Datenpflege in Alma

DOI: https://doi.org/10.31263/voebm.v73i1.3417

(C) Mathis Kronschläger

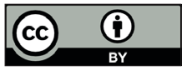

Dieses Werk ist - exkl. einzelner Logos und Abbildungen - lizenziert unter einer Creative-Commons-Lizenz Namensnennung 4.0 International 


\section{Für jeden Anlass das passende Layout - Die Verbundsuchmaschine im neuen Design}

Der Relaunch der Verbundsuchmaschine (https://search.obvsg.at) brachte neues Design und neue Features für die Recherche in den Beständen der über 90 Einrichtungen des Österreichischen Bibliothekenverbundes.

Mit dem überarbeiteten Design gestaltet sich die Suche in der mehr als 13 Millionen Titel umfassenden Verbunddatenbank noch einfacher. Dank des neuen responsiven Webdesigns wird die Benutzungsoberfläche optimal an das Layout des Bildschirms angepasst und ermöglicht eine unkomplizierte Recherche vom Smartphone, Tablet oder Computer aus.

Der Bereich „E-Ressourcen im Verbund“ ergänzt die Suchbereiche „Fachliteratur", „Hochschulschriften“ und „Nachlässe / Handschriften“. Das neue Feature erleichtert die gezielte Suche nach von den Verbundbibliotheken lizenzierten elektronischen Dokumenten wie E-Books oder EJournals.

Der Relaunch brachte wesentliche technische Verbesserungen. Auf Basis der neuen Technologie wurde im November der Bereich „Sammlungen“ vorgestellt. Dort ist der Bereich „Nachlässe und Handschriften“ verzeichnet und übersichtlich durchsuchbar.

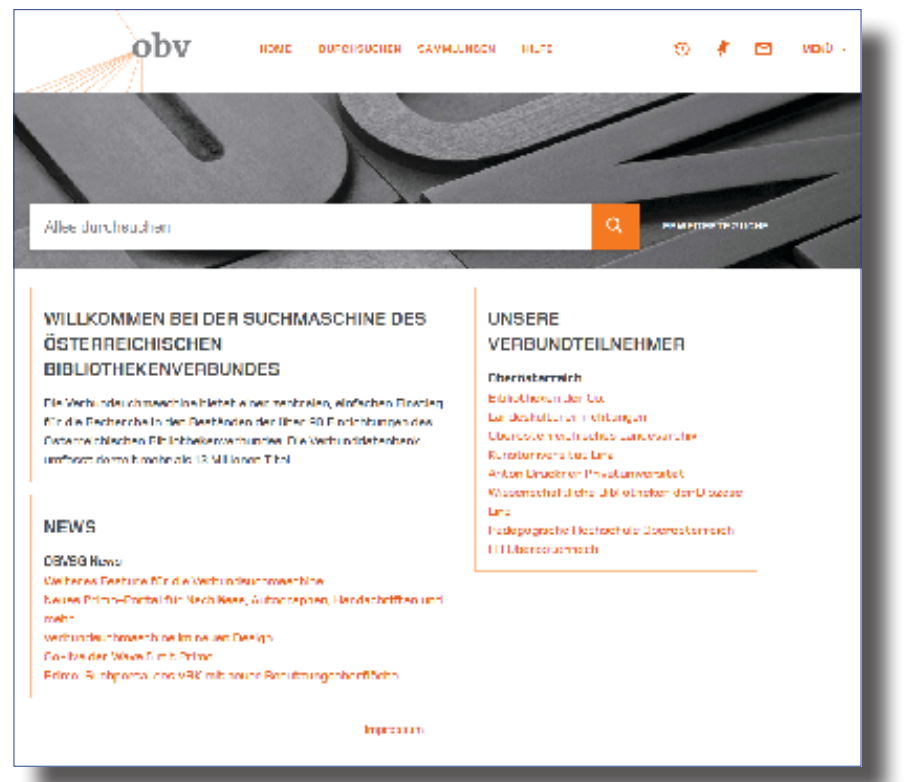

Abb. 1: Die Verbundsuchmaschine im neuen Design 


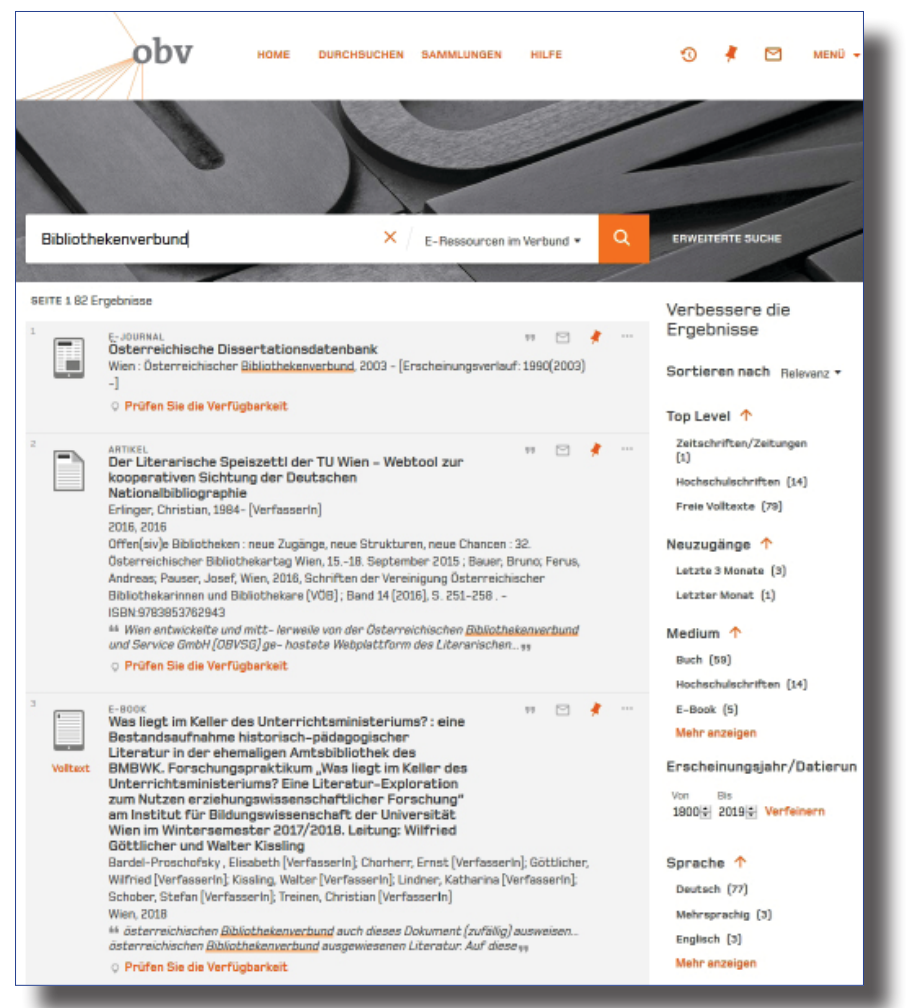

Abb. 2: Eine Recherche nach E-Ressourcen im Vebund

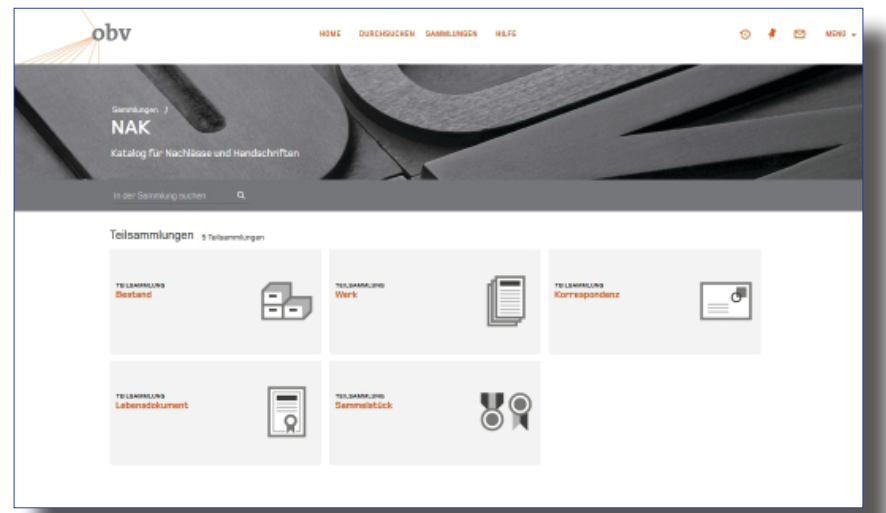

Abb. 3: Sammlungen in der Verbundsuchmaschine: der Katalog für Nachlässe und Handschriften 


\section{Grüne Welle für Alma - Aktuelles zur Alma-Implementierung im OBV}

Acht weitere Bibliotheken haben den Systemumstieg geschafft: Die Wave 5 ist seit Anfang September live mit Alma und der Produktionsstart der Wave 6 folgt im Jänner 2020. Die Bibliotheken der Wave 5 sind die:

- Akademie der bildenden Künste Wien

- Donau-Universität Krems

- Fachhochschule Salzburg

- FH Campus Wien

- FH Campus 02

- FH Wien der WKW

- Paracelsus Medizinische Privatuniversität

- Oberösterreichische Landesbibliothek mit den weiteren Landeskultureinrichtungen

Ein Systemwechsel dieser Art ist eine Herausforderung, die nur gemeinsam zu bewältigen ist. Die OBVSG hat bei der Wave 5 in enger Zusammenarbeit mit den Bibliothekarinnen und Bibliothekaren vor Ort für sechs der acht Bibliotheken den Systemwechsel durchgeführt. Das beinhaltet die Projektkoordination mit der Herstellerfirma, Hilfestellungen beim Erarbeiten neuer Geschäftsgänge, Datenanalysen und -korrekturen, Schulungen und vieles mehr.

Die Einrichtungen der Wave 6 stehen kurz vor Produktionsstart: ihr Golive wird Anfang Jänner 2020 stattfinden.

Für Bibliotheken, die im Modell Aleph- Sharing, bzw. im Modell AlmaASP von der OBVSG betreut werden, gibt es mit der Wave 6 eine Neuerung. Erstmals übernimmt die OBVSG bei den meisten dieser Einrichtungen zusätzlich zu systembibliothekarischen Aufgaben die operative Migration der Daten vom alten ins neue System. Vom dafür erworbenen Wissen, beispielsweise über das performante Laden großer Datenmengen mittels der AlmaSchnittstelle, können auch neue Verbundteilnehmer in Zukunft profitieren.

Die Wogen glätten sich noch nicht: Im Oktober hat für die Wave 7-Bibliotheken die Onboarding-Phase begonnen. Auch 2020 wird wieder ein bewegtes Jahr in Sachen Alma.

\section{Wissen vermehrt sich, wenn man es teilt - Der Digitale Assistent startet im Verbund}

Die OBVSG startet einen neuen Service. Der Digitale Assistent wird ab 2020 die Sacherschließerinnen und Sacherschließer bei ihrer Arbeit unterstützen. 
Der Digitale Assistent ist ein webbasiertes Vorschlagstool zur Unterstützung der intellektuellen verbalen und klassifikatorischen Inhaltserschließung. Er ermöglicht es, im Verbundkatalog, in den Katalogen wichtiger Partnerverbünde und bei weiteren großen Datenanbietern wie der Library of Congress schnell und unkompliziert Vorschläge für die ErschlieBung der eigenen Ressourcen zu finden.

Bei seiner Präsentation am Verbundtag und am Österreichischen Bibliothekartag ist das neue Tool auf reges Interesse gestoßen. In der Zwischenzeit wurde der Digitale Assistent durch Expertinnen und Experten der Zentralen Redaktion Sacherschließung umfangreichen Praxistests unterzogen. Gemeinsam mit der OBVSG wurde ein Setup für den Verbund erarbeitet. Nach sorgfältigen Anpassungen des Datenformats an die Standards des Verbunds stehen im OBV-Profil alle relevanten Felder der Inhaltserschließung zur Verfügung. Für die verbale Inhaltserschließung beispielsweise RSWK-Folgen, Formschlagwörter und GND-Einzelschlagwörter; für die klassifikatorische Inhaltserschließung die Basisklassifikation, die Regensburger Verbundklassifikation oder die Dewey Decimal Classification.

Weitere Informationen zum kostenpflichtigen Dienst finden Sie demnächst auf der OBVSG-Website.

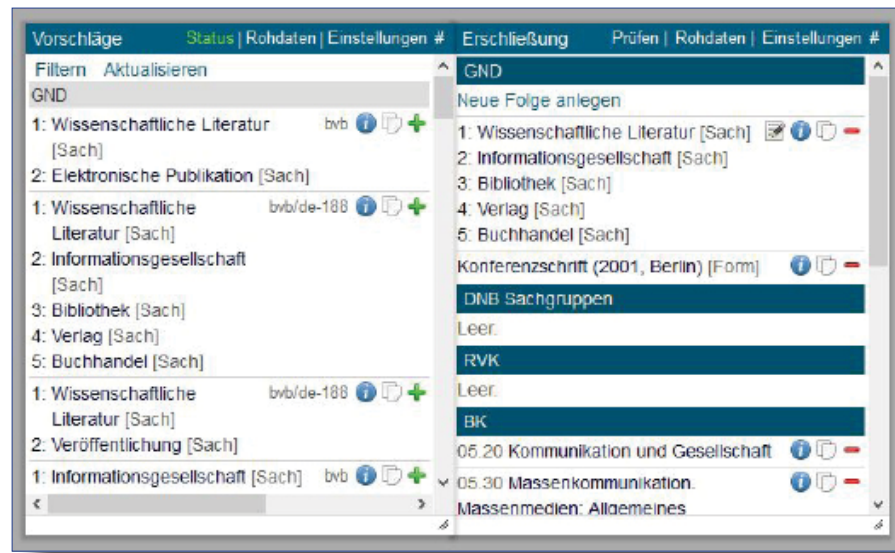

Abb. 4: Detailansicht - Datenübernahme mit nur wenigen Mausklicks

\section{Save the Data - Ausschreibung eines konsortialen Systems zur digi- talen Langzeitarchivierung}

Die Österreichische Nationalbibliothek und die OVBSG suchen in einer gemeinsamen Ausschreibung nach einem System zur digitalen Langzeitar- 
chivierung, das ab 2021 auch weiteren Einrichtungen zur Verfügung stehen soll.

Der Bedarf nach einer Lösung zur Langzeitarchivierung ist bereits seit einiger Zeit ein vieldiskutiertes Thema im Verbund. Nach mehrmonatigen Vorarbeiten konnte die Ausschreibung im September veröffentlicht werden. Juridisch begleitet wird das „EU-weite Verhandlungsverfahren mit vorheriger Bekanntmachung" durch eine auf Vergabeprojekte spezialisierte Kanzlei. Die fachlichen Vorgaben wurden gemeinsam durch die Österreichische Nationalbibliothek und die OBVSG erarbeitet. Mit einem Abschluss des Verfahrens bis zum Sommer 2020 kann gerechnet werden.

Bei den Anforderungen an das neue System liegt der Schwerpunkt auf Bibliotheksmaterialien. Anderes Material ist nicht ausgeschlossen, solange die einschlägigen Standards der elektronischen Archivierung dafür anwendbar sind. Die endgültigen Spezifikationen werden im Zuge des Verfahrens ausverhandelt.

In bewährter Verbundmanier wird die neue Software als Konsortiallösung konzipiert. Die OBVSG wird die Langzeitarchivierung als neuen Verbundservice anbieten - mit der Österreichischen Nationalbibliothek als erster Mandantin. Teilnehmende Einrichtungen können dabei sowohl von der langjährigen Erfahrung der OBVSG als Servicegesellschaft als auch von Synergieeffekten auf technischer Ebene profitieren.

Neben Mitgliedseinrichtungen des OBV soll die Lösung auch für österreichische Archive und Kulturinstitutionen des Bundes angeboten werden.

\section{GND-Datenpflege in Alma}

Datensätze der Gemeinsamen Normdatei GND stehen seit dem Produktionsstart von Alma für die Erschließung von Ressourcen zur Verfügung. Jetzt ist auch die Pflege der GND-Daten in Alma möglich.

Die Normdatenpflege, sprich die Eingabe von neuen und die Bearbeitung bestehender GND-Datensätzen in Alma war bereits für die Zeit kurz nach Go-live des neuen Systems geplant. Die aktive Mitarbeit an einer Normdatei, die in einem externen System gehostetet wird (GNDMasterfile an der DNB), war für Alma jedoch Neuland. Es mussten erst zahlreiche Probleme entsprechend der Vorgaben der OBVSG behoben werden. Seit diesem Sommer stehen alle nötigen Funktionen zur Verfügung und nach der Schulung der Kolleginnen und Kollegen der Kohorten bzw. Waves 1 bis 5, konnte die produktive Phase im November endlich beginnen. 
In Alma gibt es deutliche Verbesserungen in Bezug auf die Aktualität der Daten. Der Alma-GND-Spiegel in der Community Zone erfährt alle sechs Stunden ein OAI-Update, während Änderungen in Aleph (ACC18) nur einmal pro Woche eingespielt wurden. Dadurch ist bei der Neueingabe von Datensätzen eine Suche in der GND-Quelldatei zur Dublettenvermeidung obsolet. Auch für diverse redaktionelle Tätigkeiten bringt die erhöhte Updatefrequenz deutliche Erleichterungen. Es gibt natürlich auch in manchen Bereichen kleinere Einbußen, beispielsweise bei der Übersichtlichkeit der Datensätze, die dem Fehlen eines Internformates geschuldet ist. Insgesamt war es das Ziel, die GND-Datenpflege in Alma so ähnlich wie möglich wie die bewährten Arbeitsabläufe in Aleph zu gestalten.

Die ACC18 steht den noch in Aleph arbeitenden Institutionen (aber nicht nur diesen) natürlich vorerst weiterhin zur Verfügung. Sie wird gemeinsam mit dem Aleph-System voraussichtlich gegen Ende 2021 abgeschaltet.

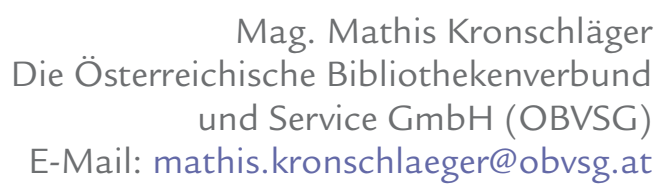

